\title{
MATH OUTSIDE THE BUBBLE
}

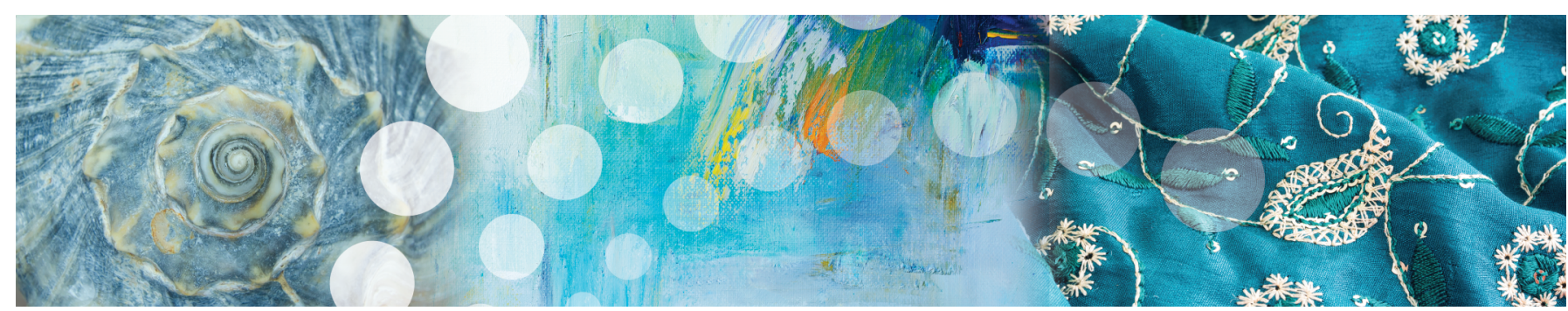

\section{"Creative Mathematician" Transforms Student Errors into "Sartorial Celebration"}

\section{Sophia D. Merow}

Life-changing inspiration struck Diarra Bousso during a late-night slog through a stack of student papers. It was 2018, and in her first few months as a high school math teacher the longtime artist had found that grading and lesson planning left her without the time-or the energy-to draw. Faced one evening with a sheaf of student attempts at graphing linear, absolute value, and quadratic functions, she began to see her pupils' misplaced vertices ${ }^{1}$ and wonky symmetries differently.

"I looked at the realm of their answers across 88 students," Bousso remembers, "and started daydreaming the patterns all their combined mistakes could produce."

The next day she pulled an all-nighter, graphing various functions and coloring the regions bounded by them. Bousso credits this wee-hours exercise with informing not only her classroom practice in the subsequent weeks but also the prints adorning her latest line of womenswear.

Bousso arrived at the overlap between parent function transformations ${ }^{2}$ and convertible jumpsuits via a

Sophia D. Merow is a freelance writer and editor. Her email address is sdmerowagmai 1.com.

${ }^{1}$ In high school math classes, the maximum or minimum point of a parabola or an absolute value function is called the vertex.

For permission to reprint this article, please contact: reprint-permission @ams.org.

DOI: https://dx.doi.org/10.1090/noti2090 multi-continent journey too long and dramatic to recapitulate fully here (but detailed by Kristen Philipkoski in Forbes in December; see https://bit.1y/2UfbW7q). Born in Dakar, Senegal, Bousso earned a bachelor's degree in applied mathematics, economics, and statistics from Minnesota's Macalester College before spending two years as a bond trader at Credit Suisse. Unhappy in finance-"I fell into depression while working on Wall Street because I had chosen the wrong path and was miserable and felt no purpose," Bousso told Forbes-she returned to Senegal in 2012, launched Dakar Boutique Group, and ran the company for five years. In 2017, she returned to the United States to pursue a master's degree in mathematics education at Stanford. There Bousso met Nomellini-Olivier Professor of Mathematics Education Jo Boaler, identified her dream job title, and began to see how pieces of her theretofore fragmented identity could in fact coexist.

When Bousso followed Boaler on Twitter back in 2017, a phrase in her then-professor's bio resonated with her: "Creative Maths." "I always struggled with my identity as both a creative and a math lover," Bousso explains. "I always felt like I had to choose one over the other and that the intersection of the two signaled confusion or just a

\footnotetext{
${ }^{2}$ High school curricula use parent function to mean the "simplest" function within a "function family." For the family of quadratic functions, for example, the parent function is $f(x)=x^{2}$.
} 
lack of focus." One focus of Boaler's work-and something she emphasizes in her classes-is visual mathematics and the brain science behind its effectiveness, and this spoke to Bousso. "She made me feel like my relationship with both math and art was valid," says Bousso. Thus validated, Bousso declared herself a "Creative Mathematician" and told Boaler she intended to make that characterization a reality.

Flash forward to last October. With her label DIARRABLU's Fall 2019 collection (see Figure 1), Bousso's math-fashion mashup came to ready-to-wear life. Colorful prints born of Bousso's paper-grading aha! splashed polygonal and curvilinear shapes across the season's jumpsuits, ponchos, and maxi dresses, and Bousso took to social media to hype her creative process. "This season we focus on quadratic equations and developed an algorithm to graph, rotate, translate, [and] dilate the geometric shapes generated by the functions," she wrote on Instagram. "We generated hundreds of prints algorithmically and picked our top 7," she tweeted.

The collection got noticed. Bousso snagged a spot as Designer in Residence at the San Francisco Fashion Incubator and in December held a Math Lab Exhibition and Christmas Pop Up at the city's Market Street Bloomingdale's. By chatting with Bousso, browsing museum-style displays of art and artifacts, and participating in hands-on activities, visitors had the chance to "follow the brand's journey from
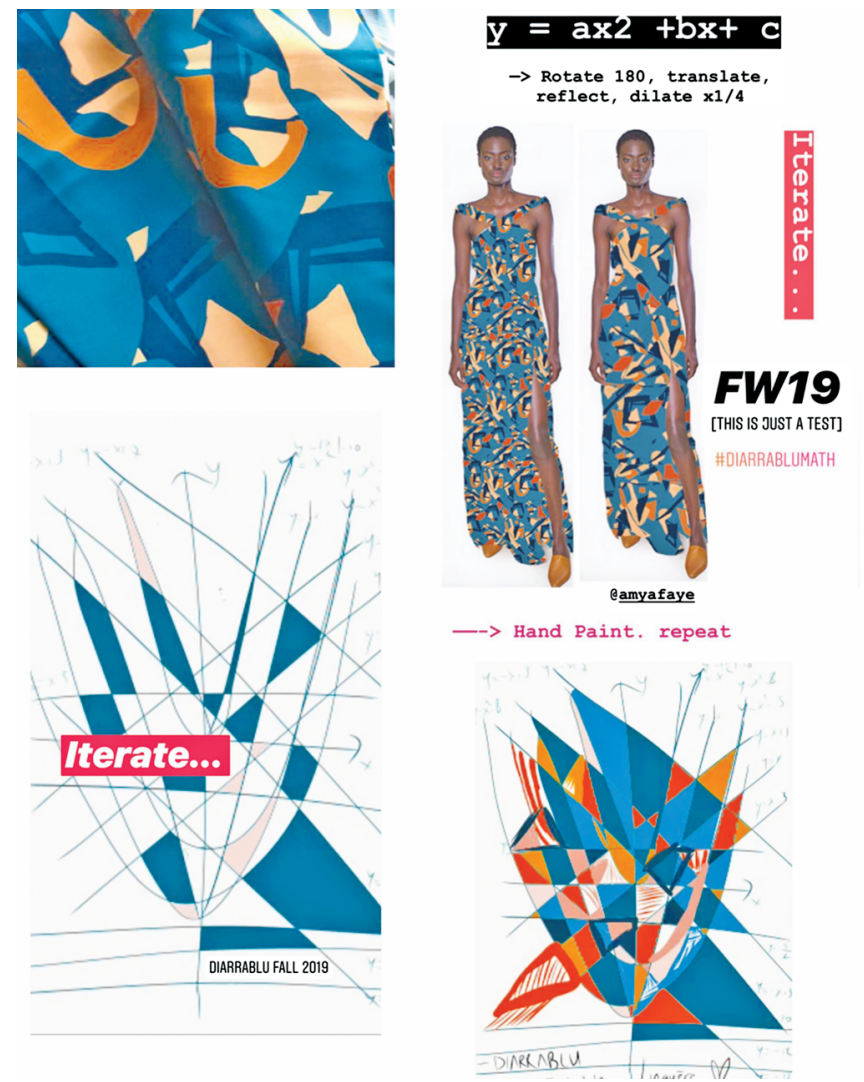

Figure 1. DIARRABLU's Fall 2019 collection signalled a new direction for the label: designing prints with mathematics.

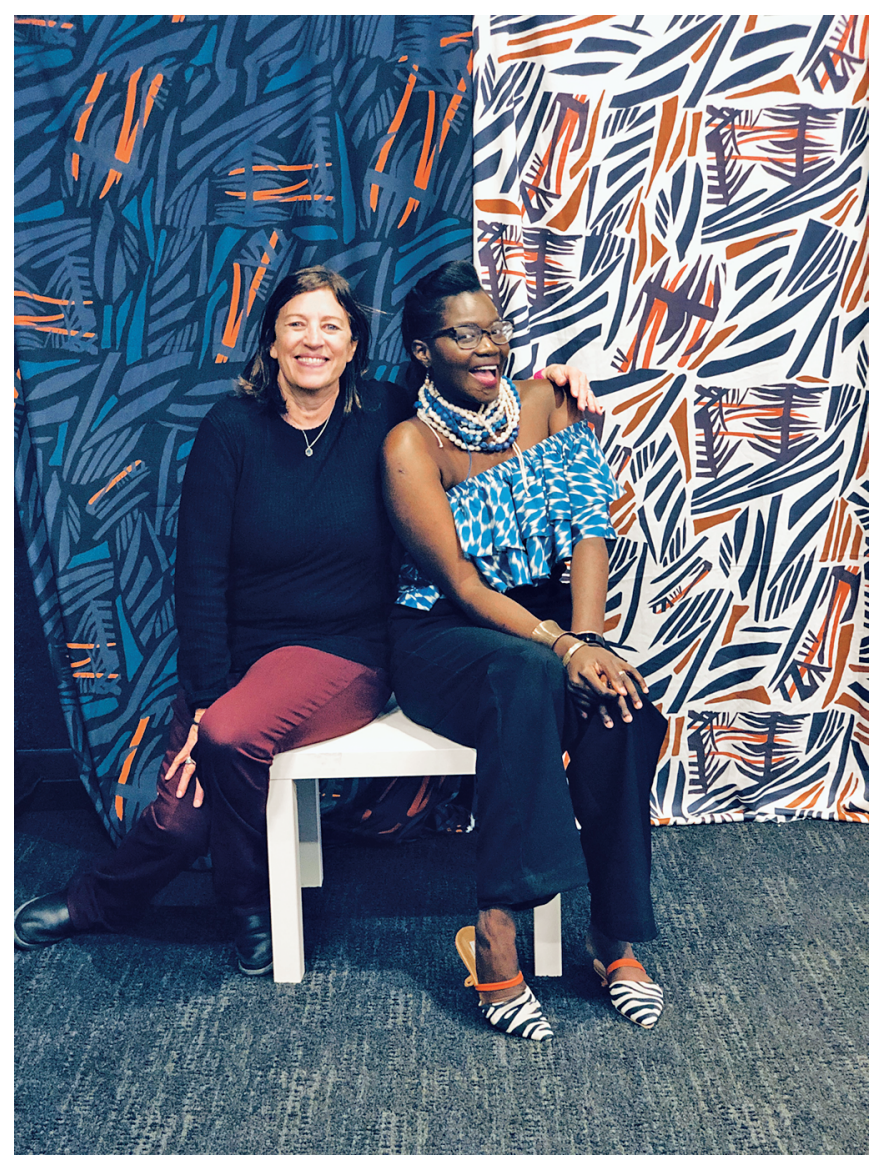

Figure 2. Boaler and Bousso at the Bloomingdale's exhibition, December 2019.

the math classroom to the garment." Fashion enthusiasts turned out, yes, but math professors (Jo Boaler, for one: see Figure 2) and students, too-a real meshing of Bousso's two worlds. As 2019-DIARRABLU's most successful year to date-drew to a close, media requests rolled in from the likes of Forbes and Vogue, CNN and Reuters. But for all the press attention, it was the engagement of her students with her work that Bousso found most gratifying. Members of Bousso's Algebra II and Geometry classes attended the Bloomingdale's exhibition and explained the math behind the designs. "It was so encouraging and humbling for me," she says.

Fresh off the initial success of her math-infused fashion, Bousso remains committed to forging ahead in both the classroom and the studio. Still teaching, she has her Algebra II students design coloring books using the same transformation process that produced DIARRABLU's Fall 2019 collection. She has collaborated with Boaler and fellow Stanford affiliate Nancy Lobell to disseminate lesson plans that equip teachers to similarly guide student exploration of "the art that can be created from mathematics" (see https://bit.1y/2v1EK62). And she has situated the mathematical element squarely at the center of her label's branding. DIARRABLU's website describes the clothing line as a "sartorial celebration of geometry and modern art," 


\section{DIARRABLU}

\section{Geometric Transformations}

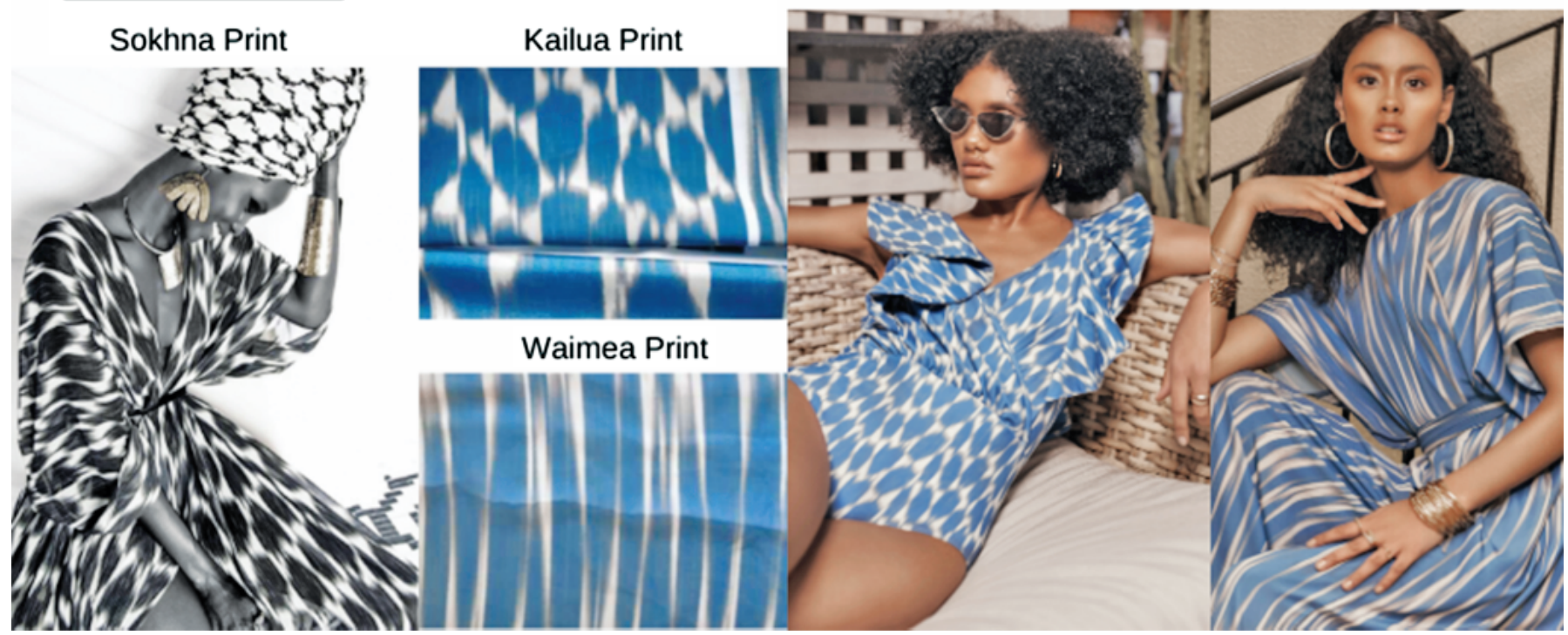

Figure 3. The Kailua and Waimea prints in DIARRABLU's Spring/Summer 2020 collection are derived from the label's bestselling Sokhna print via dilation.

one that results from "an innovative celebration of math concepts such as Euclidean geometry, combinatorics and recursion."

Pursuing mathematics education and fashion such that each informs the other has been personally fulfilling for Bousso. She can own both her mathematical and artistic interests, but also-she hopes-help others feel the liveliness of mathematics and appreciate it as a creative discipline. "Equations are not just variables and numbers, they are emotions, colors, stories, shapes," she says. "They are magic!"

Asked what readers can expect from DIARRABLU going forward, Bousso promises "a lot more math magic" and "more exploration of math art from other fields beyond algebra and geometry." Follow Bousso (@dbdiarrabousso) and/or DIARRABLU (@thediarrablu) on Twitter to stay in the loop.

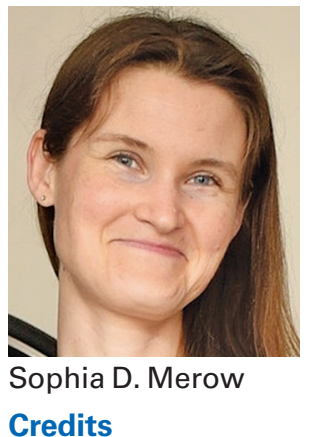

Figures are courtesy of Diarra Bousso. Author photo is by Igor Tolkov. 